\title{
Healthcare Integrated Approach as a Dynamic Process leading Innovation and Inclusive growth: Conceptual Framework based on Care Puglia (Italy)
}

Rosa Orlando ( $\square$ rosa.orlando@unisalento.it)

Universita del Salento https://orcid.org/0000-0002-7097-5431

Carmine Viola

Universita del Salento

Marco Benvenuto

Universita del Salento

Research article

Keywords: Health Service, Socio-Economic Impact, Health Economics, Chronic Care, Integrated Care, Sustainability

Posted Date: May 21st, 2020

DOl: https://doi.org/10.21203/rs.3.rs-24822/v1

License: (c) (i) This work is licensed under a Creative Commons Attribution 4.0 International License. Read Full License 


\section{Abstract}

Background Societal challenges introduced by Horizon 2020 and global challenges around health and environment expressed by seventeen sustainable development goals seek a mission-oriented agenda. Healthcare evaluation require a portfolio of projects with bottom-up experimentation. Current economic evaluations do not include a social component within the analysis. This study describes an integrated care model involving several stakeholders across healthcare sectors primary, secondary, tertiary, private and community sectors. The new integrated care framework has the aim encompass social welfare.

Methods The existing evidence about the economic impact of integrated care available in the thin scientific literature is inconclusive. A European programme called Strategic Intelligence Monitor on Personal Health Systems Phase 3 presented 24 projects of integrated care service. Those studies were set in fourteen different European countries at exception of Israel and USA. Those projects included telehealth, telecare and independent solutions for older patients with chronic conditions. Those studies mainly identified drivers and barriers of the organizational models to integrate healthcare and social care, they stress the role of Information and Communication technology in facilitating the integration of healthcare and social care.

Results Care Puglia based on Assisted Diagnostic \& Therapeutic Pathway (PDTA) developed a chronic care clinical network in the southern region of Italy. This study is going to implement a Situational Awareness Team (SAT) with the ability to fuse different types of information generating a real-time sense-check and understanding barriers to change while unfolding the properties of the model. The new framework is going to focus on the Socio-Economic aspect of the Integrated Care system fostering Management Performance, Cost-Effectiveness Analysis and Sustainability Plan. A well-informed local health authority system counts of better transparency upon local governance. The mainstay of this new integrated approach to healthcare is engaging the process of building organisations to becoming learning organisations. Integrated care results being a dynamic approach to the assessment of health policies to replace the more static cost-benefit approach. This pillar is about a smart thinking allowing innovation and inclusive growth.

\section{Background}

Health care systems are facing the challenge of efficiently meeting the complex care needs [1]. The rapidly increased prevalence of chronic disease requires long-term care and bring together a broad range of professionals, who integrate and coordinate services along the continuum of care. So, health service will be transformed over the next decade [2], to better meet long term care for chronic patient and staff needs. Health service delivery aims to invest on out-of-hospital care, prevent health inequalities, offer more digital solutions, upgrade technology and improve care quality and outcomes.

We often refer to the Healthcare System as one institution, but it is very fragmented, with Primary, Secondary, Social and Health Community care. Steps must be taken to vertically integrate secondary 
care, as well as horizontally speeding up primary and community integration. The new healthcare perspective deviates substantially from simple health technology assessment.

Expanded Chronic Care Model perspectives, developed across different sites in Europe [3], addresses integrated care challenges through two different emphases: 1) improving local stakeholder communications and collaborative processes, and 2) direct improvement in care delivery systems and tools [3]. This study is going to build a socio-economic integrated-care model that enables a technical discussion at community level using community-based resources, this model proposes an intervention called "Situational Awareness Team".

Situational awareness is an essential component of a well-integrated system and the team is expected to smartly fuse different types of information and generate a real-time sense-check; a sort of collective assessment and clever intuition on hurdlers and pitfalls which hinder a well-functioning health integratedsystem. This framework does provide foundation for Management Performance, Decision Analysis and Sustainability Plan of Care Puglia Integrated-Care System.

This study is proposing two main objectives 1 ) a socio-economic evaluative framework based on results of chronic care model developed by Puglia Care [4] and 2) setting performance indicators to analyse best practice standards pursuing sustainability.

In order to have a health system fitting the complex package of interventions with a big spectrum and comprehensive scope of care, this model is going to develop the base for a well-functioning "Situational Awareness Team" identifying its roles and duties. Key role of the team is informative of an integrated care plan for continuum of care of preventive and curative services, according to the needs of local authority. The key duty is supporting harmonization of a well-functioning integrated system in its different dimensions: health promotion, disease prevention, diagnosis, treatment, disease-management, rehabilitation and palliative care services, through the different levels and sites of care within the health system [5].

Management Performance will build, on a standardized secondary care pathway, a primary \& community care PDTA pathway (Assisted Diagnostic and Therapeutic Pathway). This pathway will establish clear data standards enabling comparison between localities and monitor performance activities, with the aim to improve services, patient outcomes and focus on efficiency. So, the evaluative framework, based on shared information of interrelated components, will permit to investigate all elements that generate certain outputs and results.

Cost effectiveness analysis, based on Time-Driven Activity Based Costing (TD-ABC), will recommend local governance, stakeholders and providers on decision making. Decision based on evidence, real data and SAT sense-check reports would define potential investment and disinvestment in resources.

Sustainability Plan will allow the system a long term sustainable economic growth. The innovative and inclusive growth should promote new jobs and strengthen local, regional and national economies [6]. 


\section{Current Economic Evaluation Methods Of Healthcare Service Delivery}

The existing evidence about the economic impact of integrated care available in the thin scientific literature is inconclusive [7]. No literature review to date presents an exhaustive methodology to evaluate and expand health economics towards integrated care [8]. Core methodology used in actual health economic framework is comparing the costs of delivering an additional unit of health outcome Health Technology Assessment (HTA) is a narrow framework and specific to one intervention outcome and it is used at local healthcare purchasing level for care services in a specific clinical scenario, it includes mainly primary and secondary settings [9] [10] [11]. It is a method with a modest impact on healthcare priority settings, its weak point is the incorporation of political and social value perspectives [12]. There is a need of finding costing methods required to extend outcome value beyond Quality Adjusted Life Years (QALYs). In fact, HTA framework is lacking in assessing community care services (social/health), patient isolation/network and tertiary/private sector. Individual stakeholders analyse opportunity costs at the margin and use this method for priority setting when considering alternative opportunities. Programme Budgeting (PB) of individual stakeholders focuses on silo budget, resource utilization, operational decision and financial management in care pathways. Budget Impact Analysis (BIA) is a similar tool with more detailed costings for individual stakeholders and measures the budget impact to provider of the new intervention or modified care pathway over a time of five-year period.

So, the current framework is a description of a fragmented system and the financial aspect of the economic evaluation is dealt at provider department level. While integrated care pathways, covering multiple care settings, are increasingly used as a tool to structure care, enhance coordination and improve transitions between care settings. However, little is known about their economic impact. Although there is evidence that integrated care can control or even reduce health care costs [4], relatively few studies have evaluated the economic impact of service delivery (SD) in integrated care models so far [5][7][8][13].

\section{Methods}

\subsection{Critical Assessment of Current Integrated Care studies of 24 European regions}

A European programme called Strategic Intelligence Monitor on Personal Health Systems Phase 3 [14] presented 24 projects of integrated care service. Those studies were set in fourteen different European countries at exception of Israel and USA. Those projects included telehealth, telecare and independent solutions for older patients with chronic conditions.

Those studies mainly identified drivers and barriers of the organizational models to integrate healthcare and social care, they stress the role of Information and Communication technology in facilitating the 
integration of healthcare and social care.

The scope of this critical assessment (Appendix A) was to identify, within the integrated system described, techniques of management performance, cost effectiveness analysis and sustainability plan.

The only study [14] that described an impact on management performance is the US largest integrated health care, this is run by Veterans Health Administration (VHA) which provides medical and social services to veterans. VHA consists of medical centres, community-based outpatient clinics, community living centres and domiciliary assistance. Every year, the Department of VHA releases a Performance and Accountability Report (PAR) along with a Performance Scorecard that comprises a summary of the most relevant results presented in the PAR. This standardised performance measurement clarifies task division, role of each board, tier of care and professionals. Cost-effectiveness Analysis and sustainability plan are not covered by this study.

A total of eleven studies mentioned some cost-effectiveness inputs, such as cost analysis, hospital admission, length of stay, emergency visits, patient's satisfaction and health related quality of life. Not full cost-effectiveness analysis has been reported.

The main inhibitors to the deployment of the integrated system, once the pilot was finished, was the lack of funding to ensure adequate resources for sustainable change and up-front costs, and the lack of innovation in reimbursement models. No sustainability measures are mentioned by the studies. There are no well-defined national investments and funding programmes to ensure adequate resources for sustainable change and up-front costs.

\section{Results}

\subsection{Integrated Care Model Explained}

The objective of this study is to build a socio-economic framework of integrated care management of service delivery. Integrated care pathway is designed for chronically ill patients with complex health problems transferring from the hospital to community services and being supervised by their general practitioner.

Health Economics (HE) modelling is mainly used for decision-making purposes and it is an essential part of healthcare planning. A systematic approach to decision making is decision-analytic modelling; economic models usually weight risk, benefits and costs of the options available. The logical mathematical framework of an economic model integrates facts and values and links these data to outcomes that are of interest to health-care decision makers. Economic decision models identify the most cost-effective intervention, the one that produces the greatest health care benefit with resources available [15].

Actual economic evaluation framework and perspective perform mechanisms that influence costs and outcomes and design a study capable of answering the questions: which interventions, for which 
patients, in which settings, using which resources?

Theories and methods should be explored in order to systematize their inclusion in the economic evaluation of integrated care and service delivery. Donabedian [16] advocated that the quality in healthcare should be evaluated in terms of "structure", "process" and "outcome". This approach can be summarised with three questions "who \& what resources", "how" and "with what result". This service delivery model encompasses several stakeholders: patients, carers, purchasers of healthcare, providers of healthcare and constituent elements. The latter are made up of inpatient and outpatient facilities and staff, which can be referred to as "service line providers".

This study is going to analyze the structure of Puglia Care chronic care [4][17][18] exploring its technical framework and identifying the functional and clinical roles of its interrelated components, and so providing development of new professional figures [19]. The idea is to set hypotheses to improve the actual model and re-framing it, in order to build an evaluative framework for performance management, decision making and sustainable planning.

After addressing the basic principles and elements of Puglia Care, this study explores two methods for costing the integration of care, those are Service Line Management (SLM) and Time-Driven Activity-Based Costing (TD-ABC). This conceptual framework of the integrated model will aim to assure costeffectiveness, efficacy and sustainability.

Service Line Management (SLM) is usually applied to improve the management (quality and productivity) of an existing intervention through a bottom-up costing analysis. Operational Research (OR) describes how services are configured and delivered, how resources should be distributed between areas, populations, programmes of care and settings; the location and accessibility of services; the size, composition and skills of the workforce; methods to improve compliance with safety and effectiveness guidelines. SLM is a process for managing the care process and aimed primarily at hospitals. This method can widen its potential opportunities for quality and productivity improvements from overlooking at clinical pathways across care settings, although can face struggles in a period of financial pressure [20].

Time-Driven Activity-Based Costing (TD-ABC) method is a more detailed approach since relies on process mapping techniques to accurately monitor all human and technology resources that contribute to a process of care [21]. It identifies resource utilization at each step of the provider team and can be taken down to individual patient level. This method is used for quality improvement, identifying which step of the process contributes to high costs and which step is delivered in efficient way. It is a technique that improves efficiency as well produces benchmarking across different sites proving the same care objectives and outcomes. TD-ABC is a technique enabling disinvestment of services which could be necessary after changes to the process of care that improve patient outcomes.

\section{2 model requirements}


Care Puglia utilized real world data from the Assisted Therapeutic Diagnostic Pathway (PDTA) database for their chronic care model. Before-after analysis of Puglia Care model shows a reduction of incidence of unplanned hospitalisation from $12.1(95 \% \mathrm{Cl}, 10.7-13.8)$ to $10.3(95 \% \mathrm{Cl}, 9.1-11.7)$ per 100 person/year. The results testify to a significantly lower number (IRR, $0.79 ; 95 \% \mathrm{Cl}, 0.68-0.91)$, length of hospital stay (IRR, 0.80; 95\% Cl, 0.76-0.84), and costs related to unplanned hospitalizations (IRR, $0.80 ; 95 \% \mathrm{Cl}, 0.80-$ 0.80 ) during follow-up in the intervention group [4].

Building up from this promising output, this study is aiming to incorporate in a socio-economic healthintegrated care framework a core team performing in a community-based environment. This team represents the "situational awareness" component of the integrated model with specific tasks and roles.

This Situational Awareness Team (SAT) works closely together on patient's Individual Care Plan (ICP) with the aim of improving clinical and functional patient's state, minimising symptomatology, preventing disability and sustaining overall quality of life. This Team is going to be responsible of suggesting on investment of community-based resources allowing the patient's access to health and social services home-based and supervising his/her social isolation. Ultimate outcome should be reducing hospitalisation and delaying institutionalisation.

Functional roles of the team are distinct and, at the same time, coadjutant. The care co-ordinator (professional nurse) works closely to the care regulator (Healthcare-Social Operator - HSO) on case by case, and both report regular follow-up to the GP, functioning as clinical leader. Each Individual Care Plan would fit into a specific health state categorisation (risk stratification) with specific care provision requirement. Mild to moderate cases are dealt by the GP and moderate to severe cases by consultant specialists. The time component in the model is an essential factor in order to monitor service provision and time series analysis of costs and outcomes (Figure 1).

The Individual Care Plan will be part of Health Educational Agenda. This is composed by two parts, patient's vadevecum (from Latin literal meaning 'come with me', concise manual with instructions) and patient's curriculum (from Latin literal meaning 'race-course', course of deeds and experiences through which people become mature), the latter describes patient's learning process. Patient's curriculum is a dynamic progress forging life-long learning competency, health literacy and social attitudes. This curriculum would report raised awareness, preventive and positive health seeking behaviour. Three main tasks of care regulator are risk assessment, formation and monitoring/evaluating chronic health state (this is part of the sustainability plan of the model) (Figure 2).

SAT guarantees access to local services network, providing health and social assistance rather than exclusively nursing. So, the socio-economic integrated care model is focused on establishing deeper local authority involvement, improve information sharing, closer working with the voluntary and community 
sector, independent sector organisations and communities. There is limited evidence of engagement with the voluntary and community sector to date, but this is planning to be the strength of the model. Care coordination and care regulation should be monitored by the SAT, providing patients with selfmanagement strategies, health coaching and social support. Chronic patient is going to feel empowered to understand and manage his/her chronic disease home based with a supported network [2].

There are needs to be addressed and significant barriers to be solved while developing a functioning socio-economic health-integrated model. SAT is expected to smartly fuse different types of information generating a real-time sense-check; a sort of collective intuition on hurdlers and pitfalls which hinder a well-functioning health integrated-system.

Figure 3 describes Socio-Economic Integrated Care Framework using Community-Based Resources (Situational Awareness Team).

This model is going to define the social and economic impact of the community based integrated model (impact assessment). Model design will try to capture common practice within a best $\&$ worst scenario. Population will be stratified by mild, moderate and high-risk chronicity, hence indicators along the pathway should describe progress of chronic condition in the pathway undertaken and foreseeable actions. The model can be used to service capacity and quality purposes (health professional workforce capacity and expertise) and cost effectiveness validation of the integrated process.

\section{3 model objectives}

Three main objectives of the model are management performance, health economics and sustainability plan (Figure 2).

\section{Management Performance - Based on Assisted Diagnostic and Therapeutic Pathway_(PDTA).}

Care Puglia is currently monitoring pathologies and its current focus is on chronicity. The implementation of Socio-Economic Integrated Care Framework will oversee Care Puglia healthcare management and financial performance.

Healthcare Resource Utilisation at sector level (such as primary, secondary and community care) should be specified, highlighting inefficiencies and adverse events including their costs and how they are reimbursed. The model would be a "real world" experience rather than a "theoretical pathway". The overall scenario should describe a cross functional team and reproduce overall outcomes at the end of the clinical condition trajectory. Little is known of chronic care management at "community level", this integrated model can incorporate factors that have an impact on patient's health state, such as isolation, patient's network at community level and tertiary sector (Figure 3). 


\section{Cost-Effectiveness Analysis - Based on Time-Driven Activity Based Costing_}

The integrated model should develop both a value proposition and a business case for adoption of a new intervention. This socio-economic model is supportive in designing new intervention and their

implementation in clinical practice. Model should specify where in the path the new intervention would fit, based on existing evidence. The intervention could develop a new service or modify an existing one. Assessing the potential cost of the new pathway, identifying resource utilisation of the revised path and QALYs (Quality Adjusted Years Gained) [22]. The methodology of the Cost-Effectiveness plan is going to set performance indicators and create standards for best practice. Socio-economic integrated-care model is going to act as an economic evaluation context that evaluates the economic and effectiveness impact of new interventions on the health system [23].

Stakeholders should define also potential impact of investment and disinvestment in resources at each sector level. Cost analysis of resource utilisation will be based on Time Driven - Activity Based Costing (TD-ABC) [24]. Decision Analysis will explore a potential method to compare complex interventions through Multi-Criteria Decision Analysis (MCDA), involving different stakeholders, considering different healthcare perspectives and scenario analyses. Decision makers of local authorities would benefit from this advanced method, which will allow to incorporate all relevant categories of outcomes and costs [25]

\section{Sustainability Plan - Based on Indicators Matrix}

This model describes chronic conditions which, by nature, are degenerative diseases.

SAT represents a determinant of sustainability enhancing an ecological public health education plan at community level, incorporating environmental and climate mediated health risks.

The newly emerging care-regulator role will adopt a holistic approach and action agenda towards sustainable ways of living at home, aiming toward sustainable health development. Care regulator would enhance and foster the desirable health seeking behaviour by promoting a greater sense of health awareness and joint responsibility. Hence, he would represent a "health-educator" raising patients' awareness, influencing their attitudes and improving overall health. Health educational agenda will include all healthy activities which empower patients acquiring skills, attitudes and essential values for personal development.

The main sustainability outcome of this framework is delaying the progressive health state degeneration. Socio-economic Integrated Care Model will design sustainability scenarios reflecting perspectives of multiple stakeholders and capturing variables at multiple levels of health delivery.

Timeframe will be reviewed collecting indicators values at multiple time points. Indicators matrix (Table 1) will monitor and evaluate the sustainability process. Short term review will indicate an organisational control, medium-long term will define behavioural change and describe impact. 
Sustainability plan is based on five indicators, measured at short, medium and long term, personal hygiene, environmental hygiene, house health \& safety, isolation (social fragility), and mobility (occupational therapy) (see table 1).

A Vector Autoregression (VAR) model will be used to measures a multivariate time series especially for describing the dynamic behavior on the use of the selected indicators. In addition to data description and forecasting, the VAR model, will be used for structural inference and policy analysis.

\section{4 implementation of integrated model}

The integrated model, by its ability to converge to common goals, create flexible functioning toward a mission led perspective. Healthcare-Social Operator (HSO) is a qualified professional figure with health and social qualifications (Puglia RR n28, $18^{\text {th }}$ December 2007). Currently the HSO acts as a hybrid figure supporting nursing and social care. Its peculiar role fits perfectly into the current healthcare domiciliary setting (see Appendix B). Puglia Region is currently forming a constant number of HSO every year $(n=127)$ with a cost of $€ 8.300$ per HSO formed per yearly.

This socio-economic framework requires an upgraded HSO figure to care-regulator role able to monitor a patient's health education agenda and individual care plan. We are going to set focus group discussions to discuss the new care-regulator role its professional functionality and formation required to explicate his functions.

Structure of the pilot model structure can be built from domain knowledge, as described by the healthcare experts, while the detailed parameter must be extracted by geo-statistical estimation techniques and validated using the available data (e.g. PDTA). The analysis is going to follow the patient's clinical pathway and his transition into different stages of illness.

Simulated model analysis is going to be implemented as a feasibility work on the territory reporting time driven integrated care activities as in figure 1. This implementation plan is going to be extended to six local health authority ( $\mathrm{Ba}, \mathrm{Br}, \mathrm{Bt}, \mathrm{Fg}$, Le and $\mathrm{Ta}$ ) as described in Appendix $\mathrm{C}$ covering Puglia Region. SAT implementation along PDTA is going to be a dynamic process supporting decision making.

\section{Discussion}

Key requirement for the use of this methodology in practice is a better knowledge and understanding of how all stakeholders involved in the care pathway can work together, both at an organizational and service level. Barriers to change should be clearly exposed in unfolding the properties of the model.

Monitoring aspect of the model is predictably easier to conceptualise and put in practice, although it requires a clear understanding of how the stakeholders involved in a care pathway can work together, both at organizational and service level. While the comparison aspect of the model should define clearly 
an appropriate return on investment from new healthcare technology, whereas clinical outcomes may take a longer timeframe to deliver. Innovation and, even, quality improvement require change in practice.

All challenges can be handled with a clear understanding of the healthcare process, top-down approach should provide informative leadership, while the bottom-up process should be led by stakeholders willingness to work together and the economic tools should translate population clinical and cost effectiveness evidence into efficient and effective local practice, providing value for money.

\section{Conclusions}

The mainstay of this new integrated approach to healthcare is engaging the process of building organisations, becoming learning organisations. Integrated care results (socio-economic evaluation outcome, performance indicators and best practice standards) are going to be baseline of the dynamic assessment process of health policies to replace the more static cost-benefit approach.

This study is proposing the implementation of SAT with the scope of developing an integrated healthcare system with a bottom-up experimentation approach.

SAT will promote communication and collaboration where it is currently lacking and dynamically weak. SAT is enhancing to provide more transparency upon local governance, although facing several challenges in spanning organisational boundaries. SAT should have problem solving abilities which lead to more thorough decision making at local level, achieving patient satisfaction (wellbeing) and healthcare organisation goals (capacity and efficiency) at the same time. Cross-functional collaboration, improved by SAT, should signalise healthcare providers with "what-is-working" and "what-is-not" at community level. A better-informed local health authority system has the potential to improve local governance across primary, secondary, tertiary, private and community sectors. Integrated healthcare system will remain a mission-oriented organisation with greater emphasis on management performance, costeffective decision making and sustainable planning.

This healthcare structure contributes in developing mechanisms so that the public and private sectors share the risks and the rewards. This pillar is about a smart thinking allowing innovation and inclusive growth.

\section{Abbreviations}

PDTA : Assisted Diagnostic and Therapeutic Pathway

SAT : Situational Awareness Team

TD-ABC : Time Driven Activity Based Costing

HTA : Health Technology Assessment 
QALYs : Quality Adjusted Life Years

BIA : Budget Impact Analysis

VHA : Veterans Health Administration

PAR : Performance Accountability Report

HE : Health Economics

SLM : Service Line Management

OR : Operational Research

HSO : Healthcare Social Operator

GP : General Pratictioner

MCDA : Multi Criteria Decision Analysis

\section{References}

[1] Payne, K, McAllister, M and Davies, LM. Valuing the economic benefits of complex interventions: when maximising health is not sufficient. Health Econ. 2013; 22: 258-71.

[2] Andria, G., Lanzolla, A. M. L., Cavallo, G., Russo, G., Marinosci, F., Incalzi, R. A., \& Benvenuto, M. A novel approach for design and testing digital $m$-health applications. In 2015 IEEE International Symposium on Medical Measurements and Applications (MeMeA) Proceedings. IEEE. 2015; 440-444

[3] Stoop A, de Bruin SR, Wistow G, Billings J, Ruppe G, Leichsenring K, Obermann K, Baan CA and Nijpels G. Exploring improvement plans of fourteen European integrated care sites for older people with complex needs. Health Policy. 2019; 123:12;1135-1154.

[4] Robusto F, Bisceglia L, Petrarolo V, Avolio F, Graps E, Attolini E, Nacchiero E and Lepore V. The effects of the introduction of a chronic care model-based program on utilization of healthcare resources: the results of the Puglia care program. BMC Health Services Research. 2018;18:377.

[5] Cowing M, Davino-Ramaya CM, Ramaya K, Szmerekovsky J. Health Care Delivery Performance: Service, Outcomes, and Resource Stewardship. The Permanente Journal. 2009;13:4

[6] Benvenuto, M., Viola, C., \& Sambati, F. A New Social and Technological Paradigm to Assess Chronic Patient Management Process: Preliminary Results. Management. 2017; 5:6; 525-540. 
[7] Nolte, E, Pitchforth, E. What is the evidence on the economic impacts of integrated care? Copenhagen: European Observatory on Health Systems and Policies. 2014 http://www.euro.who.int/_data/assets/pdf_file/0019/251434/What-is-the-evidence-on-the-economicimpacts-of-integrated-care.pdf Accessed 22 January 2020.

[8] Tsiachristas A, Stein K.V, Evers S. and Rutten-van Mölken M. Performing Economic Evaluation of Integrated Care: Highway to Hell or Stairway to Heaven? International Journal of Integrated Care. 2016; 16:(4) $3,1-12$.

[9] Drummond, MF, Sculpher, MJ, Torrance, GW, O'Brien, BJ \& Stoddart, GL. Methods for the economic evaluation of health care programme. Third edition. Oxford: Oxford University Press. (2005)

[10] Scotland G, \& Bryan S. Why Do Health Economists Promote Technology Adoption Rather Than the Search for Efficiency? A Proposal for a Change in Our Approach to Economic Evaluation in Health Care. Medical Decision Making. 2016; 37: 139-147.

[11] Goodwin, E \& Frew EJ. Using programme budgeting and marginal analysis (PBMA) to set priorities: reflections from a qualitative assessment in an English Primary Care Trust. Social Science \& Medicine. 2013; 98: 162-168.

[12] GECES - European Commission Expert Group on Social Entrepreneurship Proposed Approaches to Social Impact Measurement in European Commission legislation and in practice relating to: EuSEFs and the EaSI. 2014 https://www.fi-compass.eu/sites/default/files/publications/proposed-approaches-tosocial-impact-measurement-in-european-commission-legislation.pdf accessed 20 january 2020

[13] Vondeling $\mathrm{H}$. Economic evaluation of integrated care: an introduction. International Journal of Integrated Care. 2004; 4.

[14] SIMPHS Strategic Intelligence Monitor on Personal Health Systems Phase 3 - (2014-2015) https://ec.europa.eu/jrc/en/simphs/integrated-care-services accessed 11 Febrary 2020.

[15] Sieber U. When should decision-analytic modeling be used in the economic evaluation of health care? EurJ Health Econom. 2003; 4:143

[16] Donabedian A. An introduction to quality assurance in healthcare. Oxford: Oxford University Press, 2003, p.200.

[17] Wagner EH, Austin BT, Von Korff M. Improving outcomes in chronic illness. Managed Care Quarterly; 1996; 4(2):12-25.

[18] Wagner EH. The role of patient care teams in chronic disease management. British Medical Journal; 2000; 320(7234):569-72. 
[19] Sellars M, Detering KM, Silvester W. Current advance care planning practice in the Australian community: an online survey of home care package case managers and service managers. BMC Palliative Care; $2015 ; 14: 15$.

[20] The Kings Fund. The NHS productivity challenge: experience from the frontline, https://www.kingsfund.org.uk/sites/default/files/field/field_publication_file/the-nhs-productivitychallenge-kingsfund-may14.pdf accessed 25 October 2019.

[21] Waago-Hansen C. How time-driven activity-based costing (TDABC) enables better use of existing resources in order to improve return on investment $(\mathrm{ROI})$ in modern healthcare and hence facilitates a sustainable healthcare system. The Health. 2014; 5: 3-8.

[22] EuroQoL group. EuroQol - a new facility for the measurement of health-related quality of life. Health Policy. 1990; 16:3 199-208

[23] Viola, C., \& Benvenuto, M. Data Envelopment Analysis (DEA) for the evaluation of Public Healthcare Structures. Electronic Journal of Applied Statistical Analysis: Decision Support Systems and Services Evaluation. 2014; 5:1, 67-82.

[24] Demeere N, Stouthuysen K \& Roodhooft F. Time-driven activity-based costing in an outpatient clinic environment: Development, relevance and managerial impact. Health Policy 2009; 92 :2-3; p. 296-304.

[25] Baltussen R, \& Niessen L. Priority setting of health interventions: the need for multi-criteria decision analysis. Cost Effectiveness and Resource Allocation; 2006; 4:14

\section{Declarations}

Ethics approval and consent to participate: Not applicable

Consent to publish: Not needed

Availability of data and materials: being a review paper, it is not required.

Competing interests: no conflict of interest

Funding: Not applicable

All authors have read and approved the manuscript

Authors' Contributions: balanced distributed the contribution among them MB, CV and RO

\section{Table 1}

Due to technical limitations, tables are only available as a download in the supplemental files section.

\section{Figures}




\section{Time Driven Integrated Care Activities}

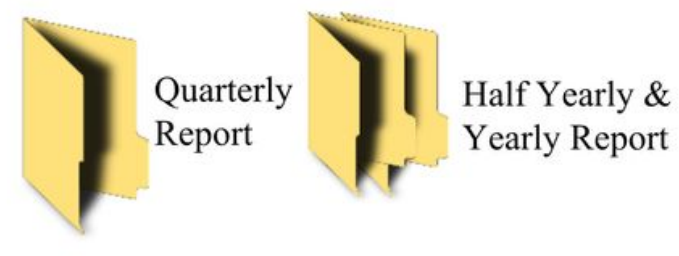

Half Yearly \&

Yearly Report

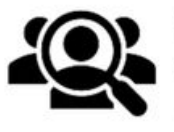

$\mathrm{SAT}^{\S}$ special review on extension activities, based on half-year and yearly report

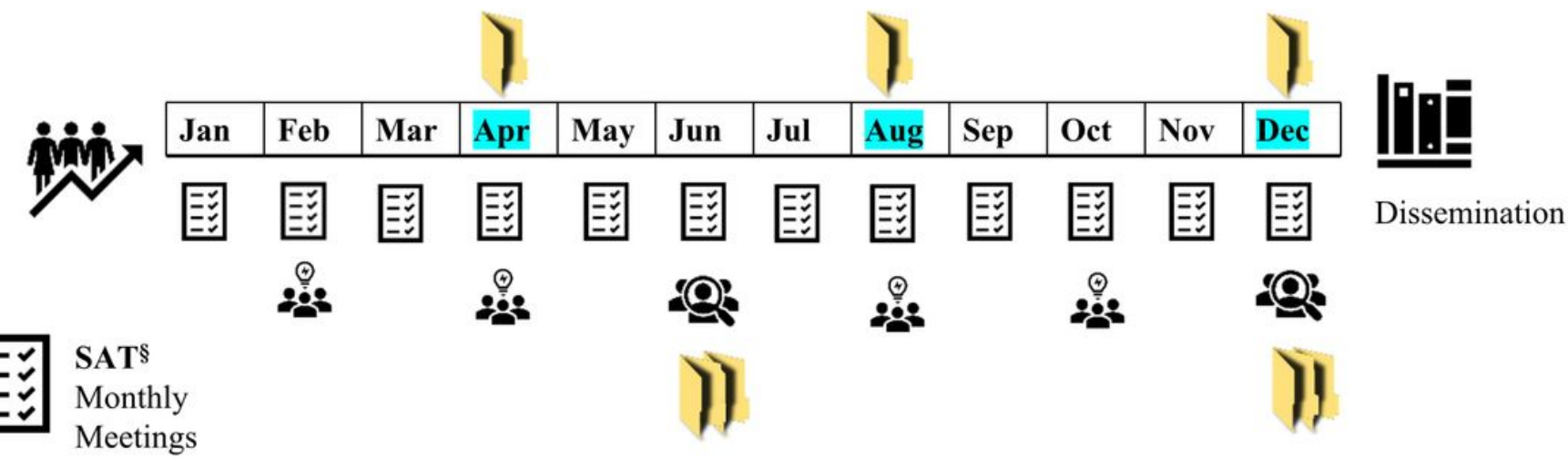

§ SAT $=$ Situational Awareness Team

SAT $\S$ updates on carecoordination, every two-month

\section{Figure 1}

\section{Time Driven Integrated Care Model}
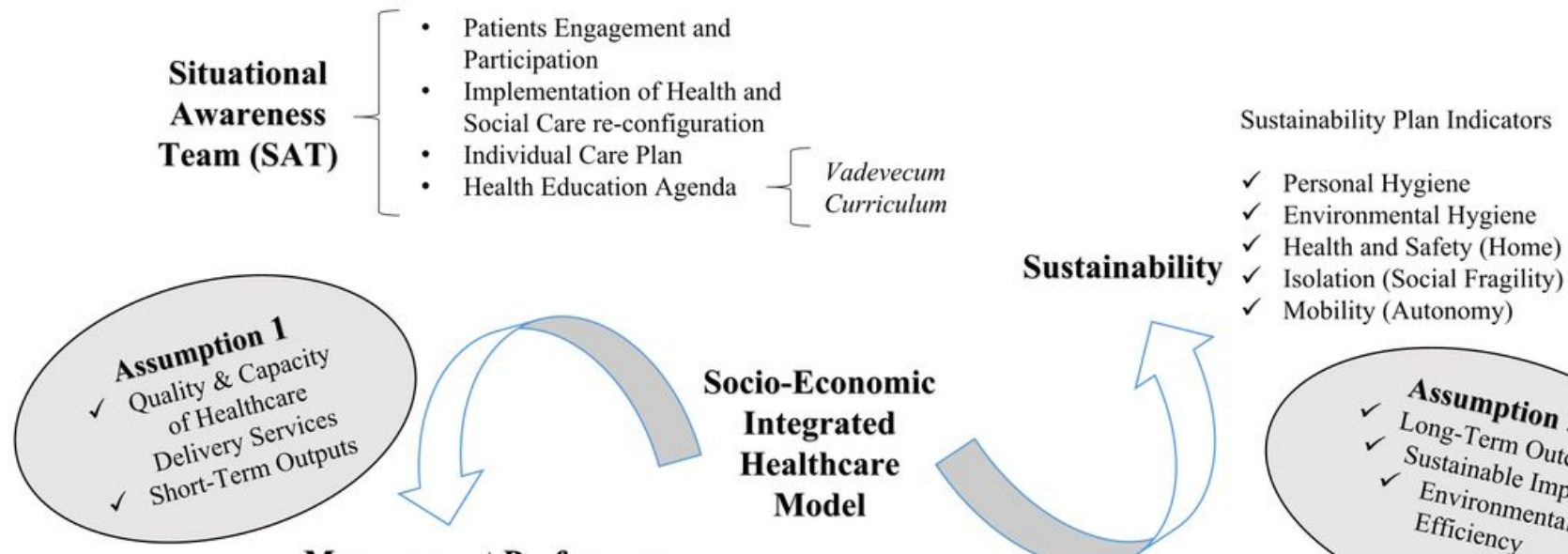

\section{Management Performance}

Operational Research

Bottom-up approach

Assisted Diagnostic and Therapeutic Pathway

Quality Performance and productivity

- Service capacity and Quality

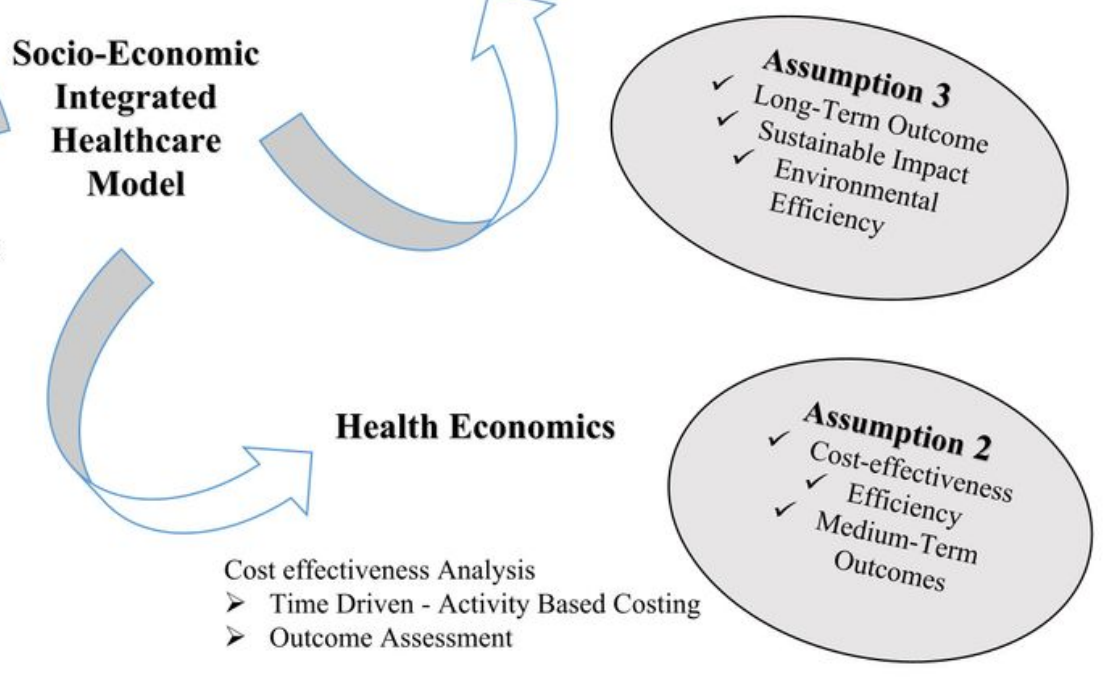


Figure 2

Integrated Care Model Objectives

Situational Awareness Team (SAT)

Clinical Leader (GP)

Care co-ordinator (Practice Nurse)

Care regulator (Socio-health operator)

Access to

Diagnostic Services

Specialist Visits

Acute Care

Timely Monitoring by SAT through Patient Medical

Record \& personal encounter with patient health and social needs

Overall Supervision

Patients access to Community Services

Health \& Social Care

Patient Interaction@ Community Level

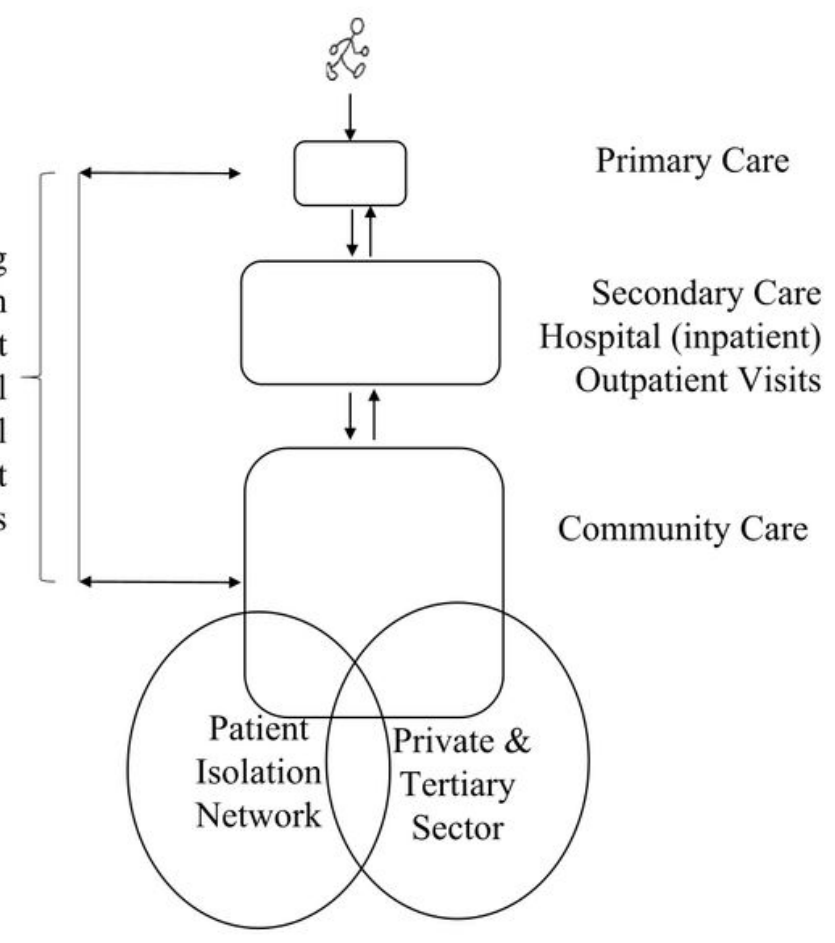

\section{Figure 3}

Socio-Economic Integrated Care Framework

\section{Supplementary Files}

This is a list of supplementary files associated with this preprint. Click to download.

- Table1.jpg 